\title{
Unexpected brain atrophy following administration of intratumoral interferon alpha-2b for cystic craniopharyngioma: A case report
}

\author{
Khadiga Elfadil Ahmed Mohammed, Kellie R Alleyne Mike, Jeannette Parkes
}

\begin{abstract}
Introduction: The use of intracystic treatments is relatively new in the treatment of craniopharyngioma and has the potential for decreased morbidity and long-term control in patients with recurrent cysts after radiotherapy as well as delaying radiotherapy treatment in young children. We describe a case in which the therapeutic use of interferon alpha-2b (IFNa-2b) was associated with an adverse effect that has not yet been documented in the medical literature. Case Report: An eight-year-old boy with a history of a craniopharyngioma was presented with radiological evidence of recurrence. He was first diagnosed at the age of eight months and had undergone repeated subtotal resections. At the age of three, he also received adjuvant radiotherapy after one of these resections. On this presentation of recurrence, a decision was made to offer him intracystic treatment with IFNa-2b. Although the typical pre-treatment leakage test was positive, the planned treatment decision was maintained following extensive multi-disciplinary consult with both local and international teams who advised that any
\end{abstract}

Khadiga Elfadil Ahmed Mohammed ${ }^{1}$, Kellie R Alleyne Mike ${ }^{1}$, Jeannette Parkes ${ }^{2}$

Affiliations: ${ }^{1}$ Senior Resident (FC RadOnc SA), Department of Radiation Oncology in Groote Schuur Hospital, Cape Town, Western Cape, South Africa.; ${ }^{2} \mathrm{Head}$ of Radiation Oncology Unit, Consultant Oncologist, Department of Radiation Oncology in Groote Schuur Hospital, Cape Town, Western Cape, South Africa.

Corresponding Author: Dr. Khadiga Elfadil Ahmed Mohammed, Contact address: South Africa, Western Cape, Cape Town, LE32 Clinic, Groote Schuur Hospital, Observatory, 7925; Tel: *27-21-404-4270; Fax: *27-21-4485707; Email: khadijaelfadil22@hotmail.com

Received: 13 June 2013

Accepted: 24 July 2013

Published: 01 December 2013 extravasation of IFNa-2b was likely innocuous. During the course of the treatment the patient demonstrated severe lethargy. Two weeks after completing the full course he presented with urinary incontinence, confusion and blood investigations confirmed hypernatremia and hyperthyroidism. Radiologic imaging showed a stable cystic mass, worsening hydrocephalus and extensive brain atrophy when compared with pre-treatment imaging. Conclusion: The potential for brain atrophy with intracystic IFNa2b has not been documented previously. While we have found no evidence to support a causal association between IFNa-2b administration and neurotoxicity, the implications of such toxicity warrant further investigation and careful observation for similar events in the future.

Keywords: Craniopharyngioma, Intratumoral interferon alpha, Brain atrophy, Neurotoxicity

$* * * * * * * * *$

Mohammed KEA, Mike KRA, Parkes J. Unexpected brain atrophy following administration of intratumoral interferon alpha-2b for cystic craniopharyngioma: A case report. International Journal of Case Reports and Images 2013;4(12):719-722.

$* * * * * * * * *$

doi:10.5348/ijcri-2013-12-419-CR-13

\section{INTRODUCTION}

Craniopharyngiomas (CPs) are of pituitary embryonic origin and are the third most common intracranial tumor of childhood; most of these tumors are cystic in nature. They are histologically benign, but can behave aggressively due to their location. Surgery has been the mainstay of treatment, but complete resection is 
sometimes impossible without undue damage to vital structures. Radiotherapy also has a role in unresectable and partially resected disease as well as recurrent disease. However, devastating side effects have been reported following both surgical resection and radiotheraphy $[1$, 2].

The long-term morbidity of patients with CPs is substantial and mainly involves endocrine, visual, hypothalamic, neurobehavioral and cognitive sequelae. This compromises normal psychosocial integration and adversely affects quality of life. The disease course is characterized by multiple recurrences prompting repeated interventions, and management decisions can become increasingly challenging as the adverse effects of earlier treatments accumulate.

\section{CASE REPORT}

An eight-year-old boy was referred by an ophthalmologist to the Pediatric Oncology Center (Cape Town) in 2004. He presented with features of hypopituitarism and bilateral optic atrophy. His imaging studies showed a ring enhancing lesion with solid and cystic components arising from the suprasellar area and compressing the third ventricle (V3) with associated hydrocephalus.

A subtotal resection and left subdural shunt was performed at that time, and a histological diagnosis of CP was made hormonal replacement was initiated.

A year later following his first recurrence the patient was treated with repeat debulking resection and at this time an Ommaya reservoir was placed. Further subtotal resection to the growing lesion, followed by adjuvant radiotherapy (54 Gy) was done the following year after he had turned three years old.

At this point, multi-modality treatment allowed for disease stability for another three years, when evidence of disease progression was again noted. This prompted surgical re-intervention and a debulking procedure was again performed.

In June 2011, at the age of eight, radiological evidence of tumor recurrence was again noted (Figure 1A-B) and after multi-disciplinary discussion, treatment with intracystic IFNa-2b was proposed as an alternative method of gaining local control and thereby controlling disease progression. A pre-treatment leakage test was done via the Ommaya reservoir and this showed extensive leakage around the catheter into the right ventricle (Figure 2). There was thus concern over the potential for leakage during IFNa-2b treatment and the clinical implications of this were researched. The treating multi-disciplinary team enlisted further advice from international colleagues regarding the safety of IFNa-2b administration in such a situation. Following this advice and the information obtained through documented research, a decision was made to proceed with the therapy as intra-ventricular spillage of IFNa was considered to be innocuous.
The first cycle was given intratumorally at a dose of 3,000,000 international units. This dose was then administered three times a week for a total of four weeks. During these treatment sessions, the patient exhibited severe lethargy. Two weeks after completing the full planned course he presented with confusion, urinary incontinence and was found to have hypernatremia and hyperthyroidism. Magnetic resonance imaging (MRI) of the brain, at that point revealed significant brain atrophy, striking volume loss with no significant change in the tumor size and an increase in hydrocephalus (Figure 3A-B).

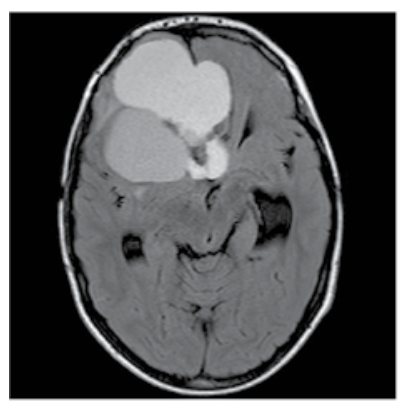

(A) Axial brain MRI

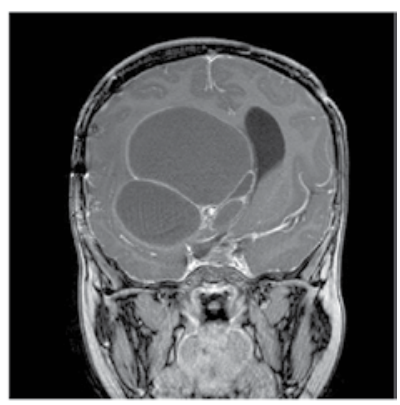

(B) Coronal brain MRI
Figure 1: (A, B) Showing the latest recurrence with a well defined, complex suprasellar mass with both cystic and solid components. Associated mass effect is noted in the ipsilateral ventricle with midline shift.

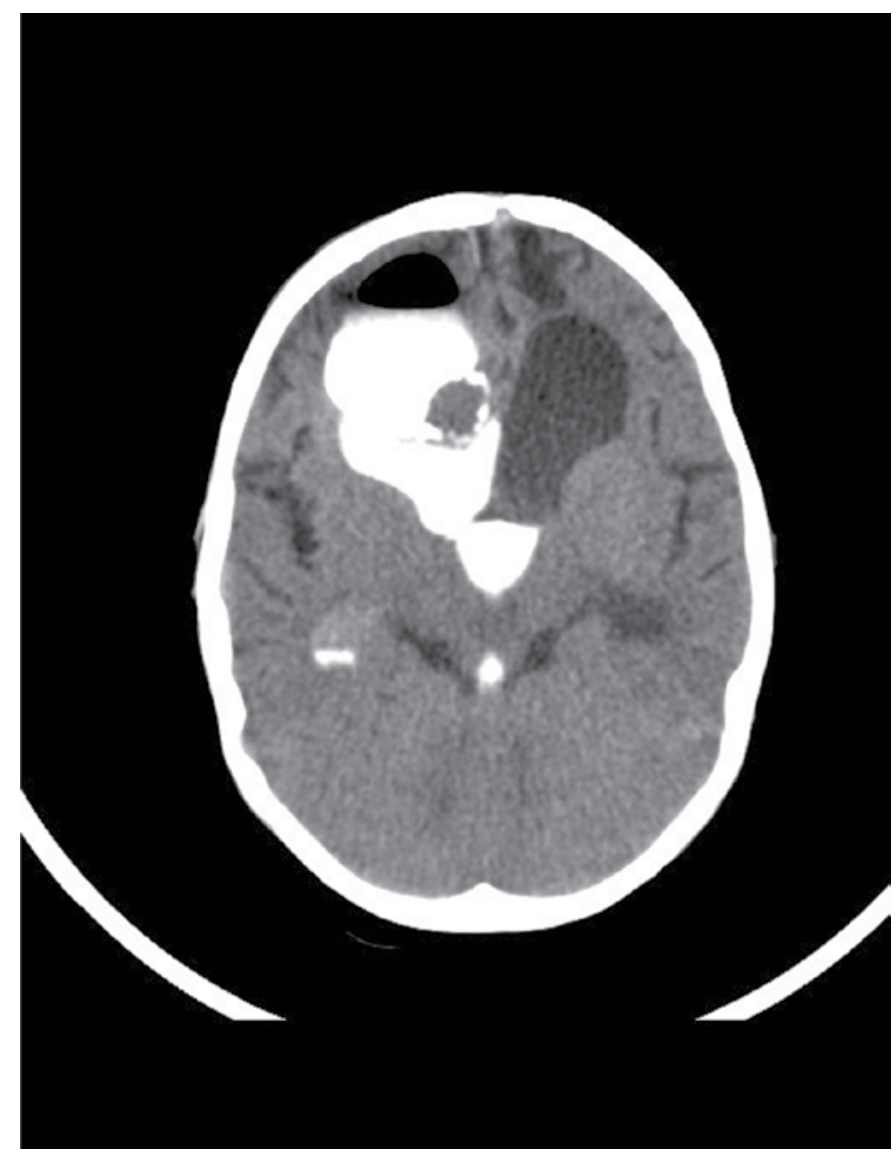

Figure 2: Computed tomography scan of the brain showed leakage of the contrast inside the ventricle during the leakage test. 


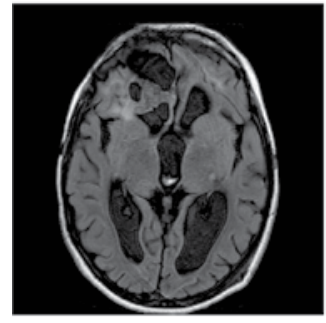

(A)Axial T1-weighted MRI of the brain

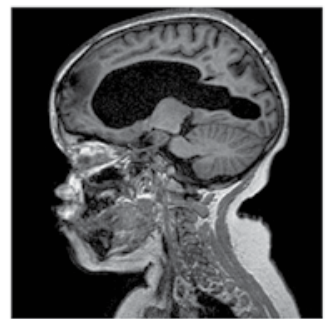

(b) Sagittal T1-weighted brain MRI

Figure 3: (A, B) Showing post treatment generalized brain parenchyma atrophy and residual tumor with associated hydrocephalus.

The patient was admitted and his condition stabilized following appropriate management. His hormonal replacement medications were adjusted and a shunt was inserted to drain the re-accumulating hydrocephalus.

He was seen again in the combined Pediatric NeuroOncology clinic a month later, and was clinically stable. It was thus decided to forgo further IFNa-2b therapy due to concern about further complications. He will thus be followed at regular intervals for symptom control and monitoring of his endocrine functions.

\section{DISCUSSION}

Craniopharyngioma is considered to be a benign chronic condition that is subject to increasing symptoms and deterioration as was observed in the current case scenario. The intractable tumor growth and treatment side effects lead to substantial long-term morbidity and impaired quality of life for these patients. IFNa has emerged as quite a promising therapy in the long-term control of the disease. It appears to be far less neurotoxic than the previously used intratumoral bleomycin, which has been fatal in some cases, and its administration requires less technical expertise than is required when using beta emitting radionuclides such as yttrium [3].

IFNa is the 1st cytokine produced by recombinant DNA for treatment of infection and malignant diseases. It has an important role in the induction of apoptosis; cell regulation and anti-proliferative effects $[1,3]$.

The first reports in the early 2000 by Jakacki et al. looked at the safety and efficacy of systemic IFNa in the treatment of CP. Tumors with a cardinal cystic component appear to be more likely to respond to IFNa than solid tumors. However, they reported significant side effects, which led to the reduction or interruption of the treatment [1].

Chamberlain et al. used IFNa as an intrathecal drug for neoplastic meningitis, and they reported both safety and feasibility of its administration into the circulating cerebrospinal fluid system [4]. The side effects were minimal and did not impede its continuation as in the case with systemic treatments.

Based on these reports, a Brazilian group treated nine patients with intracystic IFNa-2b, empirically; 3,000,000 units of IFNa were injected intratumorally, three times a week for four weeks [5]. The results were impressive, with all the patients experiencing some degree of response and reduction of the tumor volume at the end of the treatment [5].

More recently the same Pediatric Neurosurgery Department, documented findings of a multicentric prospective study in which they used intracystic IFNa in 60 patients. The same doses were employed and the presence of leakage was not considered a contraindication for the treatment as there was no concern over neurotoxicity. In this study $78.3 \%$ of the patients obtained tumor control but $31.3 \%$ exhibited new endocrine dysfunction [6]. Of note is that $30 \%$ experienced side effects (headache, palpebral edema, fever, chronic fatigue syndrome and arthritis), though this did not prevent the continuation of the treatment.

Another retrospective study was undertaken to further assess the effectiveness of intratumoral IFNa in cystic CP [7]. Nineteen patients were reviewed, and in this study there was also variable degree of tumor reduction with treatment being well tolerated and no fatalities associated with its administration.

Side effects commonly attributed to IFNa administration are transient and include chemical arachnoiditis, chronic fatigue syndrome, fever, loss of weight, loss of appetite and behavioral changes. However, no major neurotoxicity has even been identified $[2,6]$.

Most centers will perform a leakage/permeability study prior to the start of intracystic treatment. Leakage can develop from increased permeability of the cyst during treatment or as a result of tumor shrinkage and subsequent catheter exposure. Hence, even in cases where the leakage test is initially negative, leakage of an intracystic substance remains a potential risk as the treatment progresses. Therefore, a non-neurotoxic intracystic medication such as IFNa presents a safer treatment option than bleomycin or radioisotopes and can achieve impressive results, as has been shown in several other patients treated in a similar manner at the same site $[2,6]$.

The leakage test was positive in our patient and he was treated with IFNa. Unfortunately, he developed cerebral atrophy following this intracystic treatment which has not been identified in any of the available literature to date.

Treatment guidelines on the management of CPs are still evolving due to the rarity of the disease and further investigation is still needed. Due to the high incidence of recurrence and the potential for significant treatment related morbidity, much still needs to be done by way of identifying innovative drugs that can change the course of this disease. 


\section{CONCLUSION}

This is the first documented case of a possible IFNa induced brain atrophy. Although a causal association between IFNa administration and neurotoxicity has not been elucidated, the gravity of the adverse effect warrants further investigation of the mechanism of occurrence and extreme vigilance for similar events in the future.

\section{$* * * * * * * * *$}

\section{Author Contributions}

Khadiga Elfadil Ahmed Mohammed - Conception and design, Acquisition of data, Analysis and interpretation of data, Drafting the article, Critical revision of the article, Final approval of the version to be published

Kellie R Alleyne Mike - Acquisition of data, Drafting the article, Critical revision of the article, Final approval of the version to be published

Jeannette Parkes - Analysis and interpretation of data, Critical revision of the article, Final approval of the version to be published

\section{Guarantor}

The corresponding author is the guarantor of submission.

\section{Conflict of Interest}

Authors declare no conflict of interest.

\section{Copyright}

(C) Khadiga Elfadil Ahmed Mohammed et al. 2013; This article is distributed under the terms of Creative Commons attribution 3.0 License which permits unrestricted use, distribution and reproduction in any means provided the original authors and original publisher are properly credited. (Please see www.ijcasereportsandimages.com/ copyright-policy.php for more information.)

\section{REFERENCES}

1. Jakacki RI, Cohen BH, Jamison C, et al. Phase II evaluation of interferon-alpha-2a for progressive or recurrent craniopharyngiomas. J Neurosurg 2000 Feb;92(2):255-60.

2. Bartels U, Laperriere N, Bouffet E, Drake J. Intracystic therapies for cystic craniopharyngioma in childhood. Front Endocrinol (Lausanne) 2012 Mar 27;3:39.

3. Steinbok P, Hukin J. Intracystic treatments for craniopharyngioma. Neurosurg Focus 2010 Apr;28(4):E13.

4. Chamberlain MC.Aphase IItrial of intra-cerebrospinal fluid alpha interferon in the treatment of neoplastic meningitis. Cancer 2002 May 15;94(10):2675-80.

5. Cavalheiro S, Dastoli PA, Silva NS, Toledo S, Lederman $\mathrm{H}$, da Silva MC. Use of interferon alpha in Intratumoral chemotherapy for cystic craniopharyngioma. Childs Nerv Syst 2005 Aug;21(8-9):719-24.

6. Cavalheiro S, Di Rocco C, Valenzuela S, et al. Craniopharyngiomas: Intratumoral chemotherapy with interferon-alpha: A multicenter preliminary study with 60 cases. Neurosurg Focus 2010 Apr;28(4):E12.

7. Dastoli PA, Nicácio JM, Silva NS, et al. Cystic craniopharyngioma: Intratumoral chemotherapy with alpha interferon. Arq Neuropsiquiatr 2011 Feb;69(1):50-5.
Access full text article on other devices

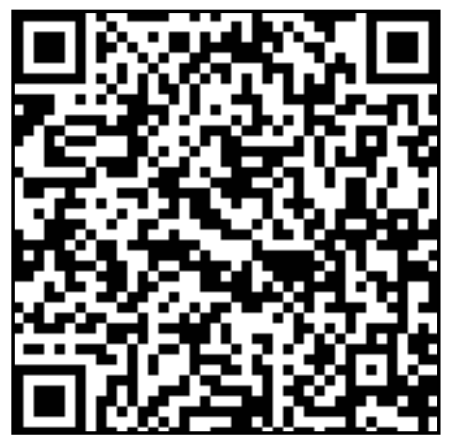

Access PDF of article on other devices

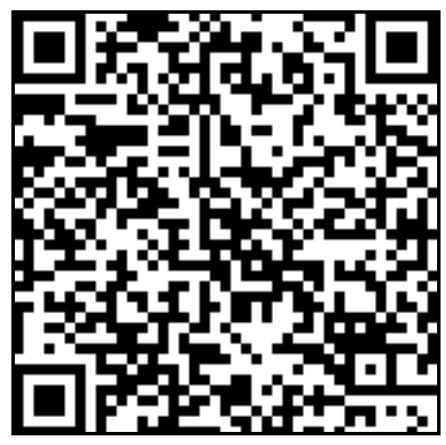

